\title{
Dynamic Test System for Generating Questions to Match Multiple Meanings in Random Order against Multiple Terminologies
}

\author{
Jae-Young Lee ${ }^{1}$ \\ ${ }^{1}$ Department of Computer Engineering, \\ Hallym University, Gangwon, Korea 200-702 \\ jylee@hallym.ac.kr
}

\begin{abstract}
In distance education, many different questions are required to evaluate the applicant's abilities or to assist effective study to learner anytime and anywhere. To satisfy requirement, we proposed the system that make question to match multiple meanings against multiple terminologies in different form whenever applicants try to take tests. For making many different questions, process in server loads terminologie and their meaning from database, and then relocates the meanings randomly using andom number before making question tests with pairs of a meaning and a terminology. The system can offermany different question tests whenever applicants try to take tests or learners, stady. Thus the system has the advantage to fairly evaluate applicants' abilifies or not to bovelearners.
\end{abstract}

Keywords: distance education, systento generate dynamic tests, automatic selection of question tests

\section{Introduction}

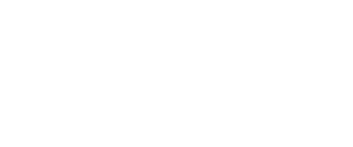

Distance education refers to as the education that enables applicants to be evaluated or learners to study anytimeanywhere. As computers, Internet, and smart phones widely spread in our lives, lots of applications have been used at every field. In the education area among various areas, manyapplications, such as, computer applications, web applications on Internet and apps for smart phone, have been developed in order to be not bored learners study and to fairly evaluate applicants abilifies. There are several researches to solve these problems in this area. As more computers had been supplied and Internet had propagated rapidly, utilizations of computer and internet had been evaluated giving positive effects on education area[1], and the distance education site based on web among them had been focused in the fields of education[2].

Distance education has been considered as an alternative education to complement space problem and quality problem that occur in a traditional education. That is, it contributes both to spread traming locations for solving the centralization problem of training locations and to supply the high quality of education. However, when evaluating the applicants' abilities anytime and anywhere, the most important thing keeps on fairness in the evaluation.

To do this in distance education, there are two groups of making question tests: automatic selection of test questions and dynamic generation of test questions. The automatic selection of test questions is the system that choices each complete question from database according to criteria that examiner had decided in advance [3-7]. Because database has complete formed questions, not components of questions, the form of chosen question is the same as that of the question stored in database. For example of multiple choice problem, question sentence, position of multiple choice items as well as their items are identical to those in the database. 
The same terms and the same position of them may have negative influence on evaluating applicants because good memory can help them with overestimating their abilities.

On the other hand, a system for dynamic generation of question tests first extracts components about each incomplete question from database which has sets of components about incomplete questions. In the next process, the question test is made by combining each question using the components. The way to combine has two: one is relocation of choice items randomly and the other is relocation of completed questions randomly[8,9,10]. This system has an advantage that offers different question tests whenever applicants try to take tests because it has lots of different question tests.

In this paper, we propose the system that make question to match multiple meanings against multiple terminologies for the above advantage. The way to accomplish the goal, specified number of terminologies and meanings is randomly loaded from database and thên meanings are relocated before making questions to match meanings against terminologies.

\section{A System to Make Question Tests}

In the system to make question tests, the system consists of chent, server, and database. The system receives information about questions from the examiner and then saves them in the database. The process at the server makes question tests and sends it to client. The client enables the applicant to take the question test. Such a clients and server system including database is shown in Figure 1.

The systems are classified as two types. One is automatie selection of question tests and the other is dynamic generation of guestion tests

In automatic selection of question test, the system-is composed of client and server with database in which the main process of the server is random choice of complete questions from database, as shownin Figure 1 . After the complete questions made by examiner are stored in database, the process in the server selects a set of suitable question from database before sending it to the client for applicant or learners. These questions are ones to meet the criteria which are specified by examiners. The questions are suitable to evaluate applicant's learning ability.

For example for automatic selection of question tests, we will explain the process of the system by three quizzes in database. The each quiz consists of one question, one correct item and three in orrect items. This quiz has immutable form because the position of four items is fjxed, that is, not movable. In the first quiz, the question is "Choose fruit?", the oosition of a correct answer is the 1st, and positions of three wrong answers are the 2nd, 3rd and 4th, as shown in Figure 2 (a) database. In the second quiz, the question is the same as the first question, the position of a correct answer is the third, and positions of three wrong answers are 1st, 2nd, and 4th. In the third quiz with the same question, the positions are 3rd for a correct answer, and 1st, 2nd, and 4th for three wrong answers. In this situation, random choice process, in the server, simply selects two quizzes randomly and makes two tests of questions. Test type A consists of quizzes 1 and 2 from database and type $B$ is made by quizzes 2 and 3 , as shown in Figure 2 (b). The important thing is that the positions of two correct answers of both same quizzes in type A and B are identical and these positions are also the same as the position of the same quiz in database. In other words, the answer of the second quiz in type $\mathrm{A}$ is identical to that of the first quiz in type B. It means that the answer of the same quiz from database is always identical without time to choose. The disadvantage of the system is to overestimate applicant's ability when the applicant tries to take the same quiz more than two times. 


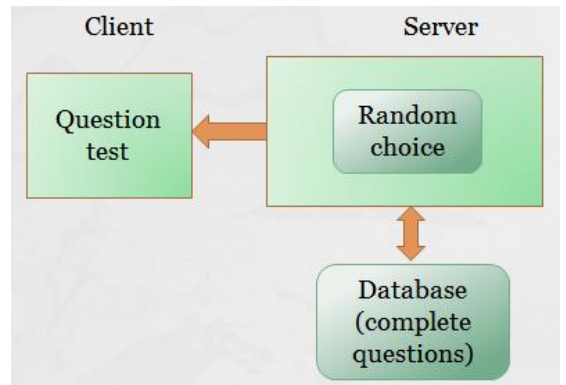

\section{Figure 1. A System for Automatic Selection of Question Tests}

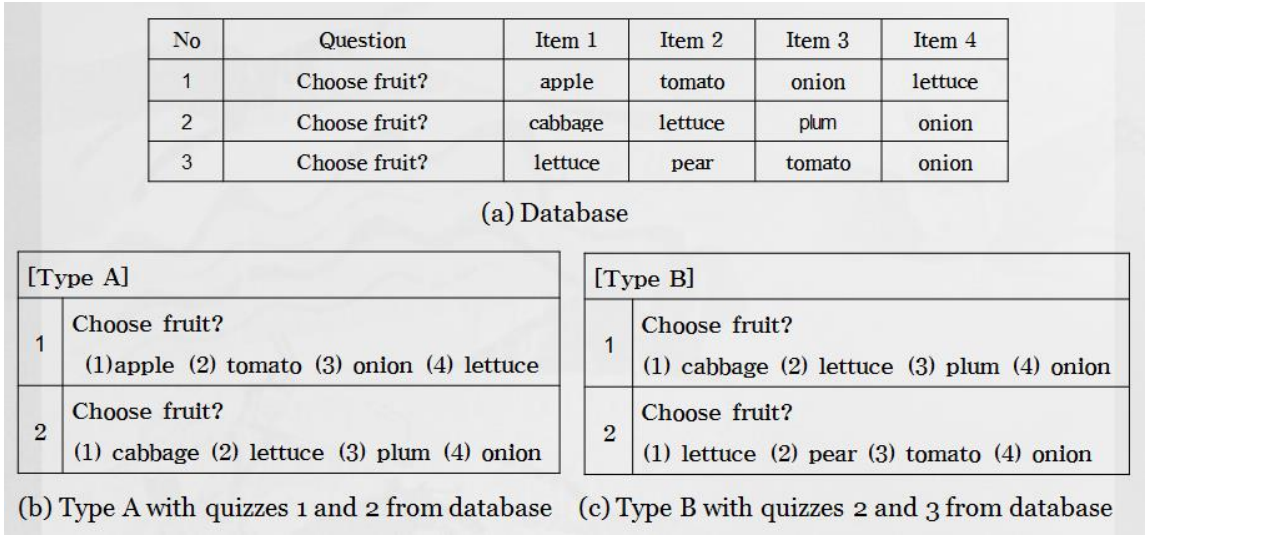

Figure 2. Example for a System for,Automatic Selection of Question Tests

In dynamic generation of question tests, the system is composed of client and server with database in which the process of random choice, in the server, is to choose components of incomplete question, the process of the first random relocation is to mix four items including a correct answer, and the second random relocation is to relocate questions with mixed items randomly, as shown in Figure 3. In this system, the components about incomplete questions are made by examiner and stored in database. After the process of random choice, in the server, selects components, such as, suitable questions, one correct item, and multiple incorrect items from database, the process of the first random relocation is to mix one correct item and multiple incorrect items, and the process of the first random relocation is to relocate mixed questions randomly before sending it to the client for applicant or learners, as shown in Figure 3. The criteria to select questions in this system can be used by that in the first system.

For example of dynamic generation of question tests, a single quiz contains components of question, such as, a question sentence, multiple correct items, and multiple incorrect items, as shown in Figure 4 (a). This quiz has mutable form because the position of four items is not fixed, that is, movable. Whenever quizzes are need for making question test, the system chooses a question sentence, and selects one of multiple correct answers and three of multiple incorrect answers before executing two relocations. This quiz has one question sentence with "Choose fruit?", three correct answers with "apple, pear and plum", and four incorrect answers with "cabbage, tomato, lettuce, and onion", as shown in Figure 4 (a). 
In the process of the random choice, the first quiz in type A contains "Choose fruit?" as a question sentence, "apple" as correct answer, and "tomato, lettuce, and onion" as incorrect answers. The process of the first relocation may changes "apple, tomato, lettuce, and onion" in selected order into "tomato, onion, apple, and lettuce" in the random order using random numbers. Thus, the answer is apple 3. The second quiz in type A contains "Choose fruit?", "plum" as correct answer, and "cabbage, lettuce, and onion" as incorrect answers. The first relocation may changes "plum, cabbage, lettuce, and onion" into "cabbage, onion lettuce, and plum" in the random order. In this case, the answer becomes plum 4 .

For another example, the first quiz of type B contains "Choose fruit?", "plum" "cabbage, lettuce, and onion". These components in the first quiz of type B are the same as those in the second quiz in type A. The process of the first relocation may changes "plum, cabbage, lettuce, and onion" into "cabbage, plum, onion, and tetuce" in the random order. The position of the correct answer in the first quiz of type B is different from that in the second quiz of type A although two positions are the same before the process of relocation. Thus, the answer in type $A$ is 4 , but the answer in type B becomes plum 2, not 4 for the same component. This means that two sets of one correct answer and three incorrect ones are identieal but two answer is hard to be the same. The probability to be the same answer is $1 / 4 !=1 / 24$ for 4 items. The process of the second relocation is randomly to change the positions of every question produced at the first random relocation.

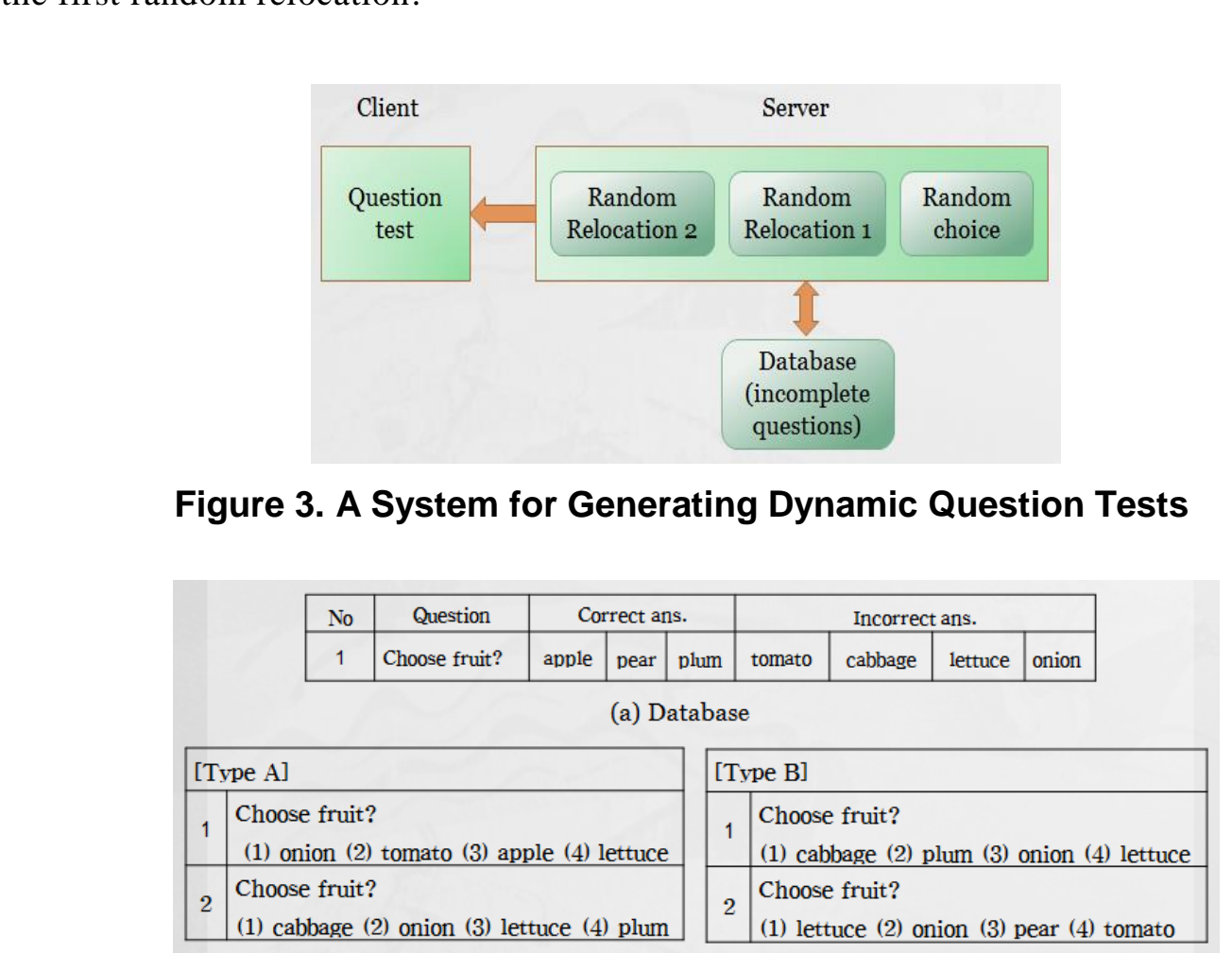

(b) Type A or B with a correct answer and 3 incorrect ones chosen from database randomly

\section{Figure 4. A System for Generating Dynamic Question Tests}

In this paper, we propose the system for dynamic generation of question to match multiple meanings against multiple terminologies which consists of client and server 
with database. There are two parts in the main process of the server. One is to choose both terminologies and meanings from database randomly and the other is to relocate one of terminologies and meanings randomly, as shown in Figure 5. The system will be explained at the next section in details.

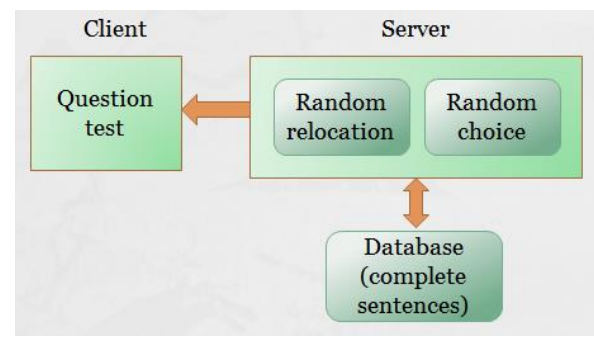

Figure 5. A System for Generating Questions to Match Meanings Against Words

\section{Algorithm for Questions to Match Using Relocation}

In this paper, we propose the client-server system to make question test to match each meaning against its terminology. The system offers different question test to applicant when he takes the test. To do this, there are three phases in the algorithm to make matching relocated meanings in random order against them terminologies.

The first phase is to extract a suitable set of terminologies and meanings from database. This set is randomly selectedup to predenned number which examiner or applicant sets. The second phase is the process to relocate one of two set: one is the set of selected meanings and the other s the set of selected terminologies. Way to match correct terminology against meaning is easier than vice versa to solve problem because the former is to search many short terminologies to choice answer after reading a long meaning one time, but the latter is to search many long meanings like sentences after reading a short meaning once. To solve problem easily, the proposed algorithm uses the former way to make question test. So components of question test consist of no, meaning, blank to write answer, and terminology. To make question test and answer table, every meaning is added number before relocating meaning, as shown in Figure 6 (a). Figure 6 (b) shows the status of the table after relocating both the numbers and the meanings except for words, which mean terminologies.

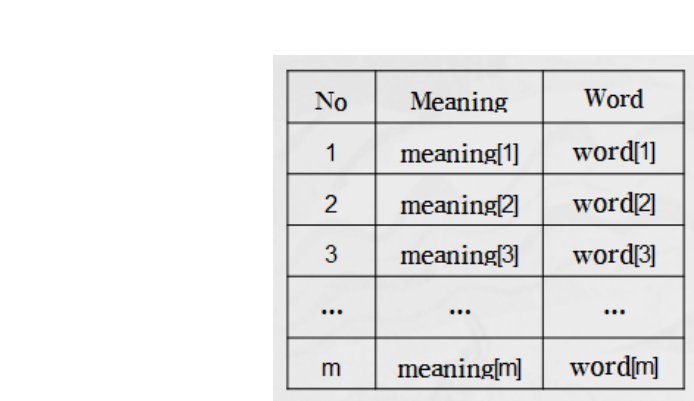

(a) Information of question before relocation

\begin{tabular}{|c|c|c|}
\hline No & Meaning & Word \\
\hline 3 & meaning[3] & word[1] \\
\hline 1 & meaning[1] & word[2] \\
\hline $\mathrm{m}$ & meaning[m] & word[3] \\
\hline$\cdots$ & $\cdots$ & $\cdots$ \\
\hline 2 & meaning[2] & word[m] \\
\hline
\end{tabular}

(b) Information of question after relocation

Figure 6. A Process to Relocate both No and Meaning Except for Word 
The last phase is the process to make question test and correct answer by separating the relocated columns into two parts: one is the set of meanings and words which are used to make question test and the other is a set of numbers which is used to make answer table, as shown in Figure 7. In this case of answer table, the first value of the table is 3 which points to the word[3]. It means that the first value of the table is the same as the answer of the first meaning[3] in question test. In the same way, the second value of the table is 1 which points to the word[1]. Thus, 1 means the answer of the second meaning [1]. We can see that $i$-th value of answer table is the answer of $i$-th meaning at the question test.

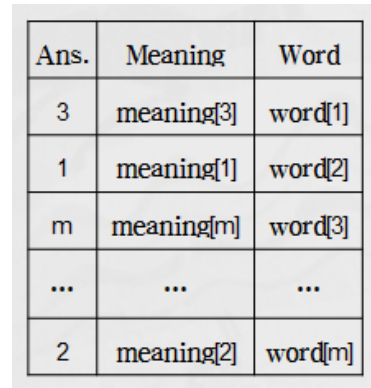

(a) Relocated Information

\begin{tabular}{|c|c|c|}
\hline No & Meaning & Word \\
\hline 1 & meaning[3] & word[1] \\
\hline 2 & meaning[1] & word[2] \\
\hline 3 & meaning[m] & word[3] \\
\hline$\ldots$ & $\ldots$ & \\
\hline $\mathrm{m}$ & meaning[2] & vord[m] \\
\hline
\end{tabular}

(b) Question test

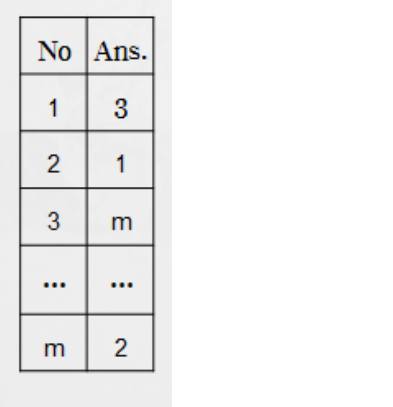

(c) Answer

Figure 7. A Process to Separate Relocated Information into Question Test and Ansyrer Table

Algorithm for making questions to match multiple meanings against multiple terminologies using relocation is the followings

[Step 1] Extract componen of questions, such as, terminologies and their meanings from database randomly up to the predefined humber.

[Step 2] if it checks the duplicate, then repeat [step 1].

[Step 3] To get answer table for scoring applicant's answers, new numbers are added in front of meanings in ascending order.

[Step 4] Initialize index pointers of the meanings.

[Step 5] To relocate both the new numbers and meanings randomly except for terminologies, repeat the process to exchange the meaning in the position of index pointer for that in the position of random number.

[Step 6] Increment the index pointer and get the random one from random function. If index is not equal to the last, then repeat [Step 5].

[Step 6] To make question test and answer table, separate relocated numbers, relocated meanings, and terminologies into two parts: one is both relocated meanings and terminologies for question test and the other is the relocated numbers for answer table.

When applicants solve the question test on the web, they cannot be satisfied the score which they get. To get better score, they may try to take the same test. To prohibit taking the previous test again, the process choices one of the terminology in the question test and sends it to the process of scoring when sending applicant's answers to the scoring process. If applicant enters his answers after solving the questions, web browser, at the client, sends applicant's answers, terminology for checking duplicate to the scoring program at the server. After receiving duplicate checking terminology and answers, the scoring process compares the terminology with the previous one. If the 
result is same, the process alerts that applicant must try to take a different test again. If no duplicate, the scoring program starts to check applicant's answers. Scoring is to compare correct answers with applicant's ones and to store applicant's score including applicant's name, pass time into record file.

Algorithm for scoring applicant's answers is the followings:

[Step 1] Receive applicant's answers, a name, and terminology for checking duplicate from the client.

[Step 2] Compare the terminology with the previous one loaded from duplicate check file. If the result is same, the process alerts that applicant must try to take a different test again.

[Step 3] Compare correct answers with applicant's ones to score applicant's ones.

[Step 4] Save applicant's name, a score, and time to pass into score history file.

[Step 5] To check duplicate next time, save current terminology into duplicate check file

When these algorithms are used to study review or preview except foreyaluation of applicant's ability, each learner can try to take test until perfect score. If the score is perfect, the perfect score is only saved into the score history file. Thus, this learning is effective in the case that every applicant's ability can be improved by the repetition.

\section{Implementations and Discussions}

The algorithm for dynamic generation of questions to match multiple meanings against multiple terminologies is implemented on environments of JSP, apache-tomcat server, file system, and mysql [11]. The system to implement the algorithm has stored terminologies and their meanings about data communications separated by chapter in database. For example, 40 terminologies and therr 40 meanings at chapter one are stored in data base. If applicant clicks the particular area which he wants to take a test, the system selects 10 terminologles and their meanings randomly and then relocates the meanings.

Figure 8 shows the screen of the question test to match meanings against terminology and blank to write answer between these two. The advantage of this system offers different question test whenever applicants try to take tests or refresh. Figure 8 shows the screen of the different question test including applicant's answers.

\begin{tabular}{|l||l||l||l||}
\hline 1. A com unio otions pathway that transfers data \\
from on device to another.
\end{tabular}

SUBMIT

Figure 8. A Screen to Show Questions to Match Each Meaning Against each Terminology 
Let's compare two question tests. Two terminologies, such as link and sender, appear both sides at two question tests, but the other terminologies are different. Figure 8 shows that the meaning of "link" is at the 1st and its terminology is also at the 1st by chance, but Figure 9 shows that the meaning of "link" is at the 7th and its terminology is also at the 4th. For "sender", Figure 8 shows that the meaning and its terminology are at the 6th and at the 7th, respectively, but Figure 9 shows the meaning at the 3rd and its terminology at the 8th.

As we saw the above, common terminologies appear fewer and positions of their meanings and terminologies are different whenever we need. This properties of this system can fairly evaluate applicant's ability and have learning effects on improving learner's ability.

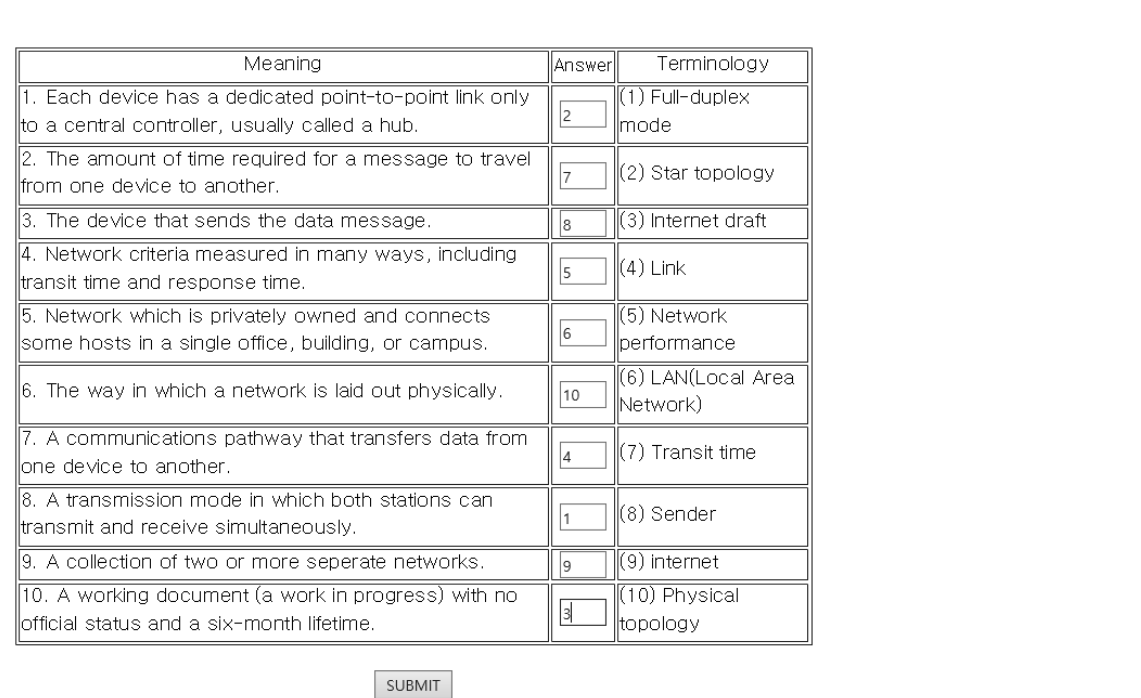

Figure 9. A Screen to Show Different Questions Including Applicant's Answers

Let us consider how many question tests the system makes. Assume that the system selects $m$ terminology sets among $n$ sets randomly $(n>m)$ and relocates the selected terminology sets. The number of different set can be represented by permutation $\mathrm{nPm}=$ $\mathrm{n} ! /(\mathrm{n}-\mathrm{m})$ !. For the example of simple calculation, if the system selects 10 terminologies from 20 ones and recates 10 ones, the number of question tests are 20!/10! = $670,442,572,800$ it means that applicants or learners can always take different tests whenever they try to.

\section{Conclasions}

In this paper, we have proposed the system that makes question tests to match multiple meanings against multiple terminologies to prevent from cheating when applicants have tests anytime anywhere or to decrease boring when learners study. To achieve this goal, the system can make money different question tests to match meanings against their terminologies, as the system randomly extracts pairs of a terminology and its meaning from database, relocates the meanings. 


\section{Acknowledgements}

This research was supported by Hallym University Research Fund, 2014(HRF-201406004).

\section{References}

[1] W. Bates, "Costing Distance Education Technologies", Open Learning Agency, (1994).

[2] A. Mary, Anytime, Anywhere Learning, Newmeia, (1997).

[3] K. A. Kim and E. M. Choi, "Automated Selection System of Estimation Questions in Web-Based Instruction", Journal of Korean Information Processing Society, vol. 9-A, no. 3, (2002).

[4] H. J. Lee, M. S. Lee, S. M. Hong, C. H. Lee and S. H. Jung, "Web-based Automatic Question-Issuing System Using Level Estimation for Learners", Journal of Korean Information Processing Society, vol. 10-A, no. $5,(\mathbf{2 0 0 3})$.

[5] E. J. Kim, "Estimation Questions Selection Algorithm in Web-based Engineer Test Educatron System", Journal of Korea Society of Industrial Information Systems, vol. 9, no. 3, (2004).

[6] E. J. Kim, "A Study on Selection Method and Mediateness Degree of Difficulty of Estination Questions in Web-based Education System", Journal of Korean Information Processing Society, vol. 12-D no. 3, (2005).

[7] C. Gutl, K. Lankmayr and J. Hofler, "Enhanced Automatic Question Creator-EAQC: Concept, Development and Evaluation of an Automatic Test Item Creation Tool to Foster Mrodern e-Educationv, Electronic Journal of e-Learning, vol. 9, no. 1 (2011).

[8] J. S. Oh, S. W. Chu, Y. S. Kim and J. Y. Lee, "A Design of Dynamic Question Generation System Using Korean WordNet", Journal of Korean Information Processing Society, vol. 4, no. 5, (2006).

[9] A. Dryver, "The Enhancement of Teaching Materials for Applied Statistics Courses by Combining Random Number Generation and Portable Document Format Files via LATEX , Journal of Statistical Software, vol. 31, no. 3, (2009).

[10] J. Y. Lee, "A System to Generate Dynamic Test Using Classes for replacing Numbers and Terms with Similar Data In Mathematical and Scientific Questions" Jounalof Korean Information Technology, vol. 4, no. 5, (2014).

[11] S. J. Guem, I. S. Kim and K. D. Choi, Tomicat, Mysql, and JSP, Daerim, (2007).
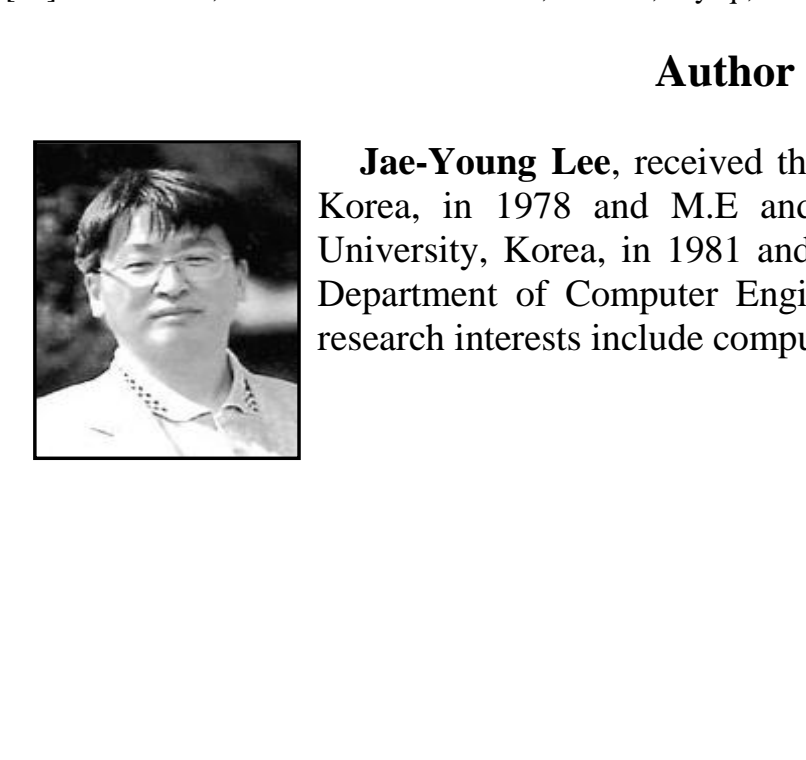

Jae-Young Lee, received the B.E. degree from Hanyang University, Korea, in 1978 and M.E and Ph. D. degrees from Seoul National University, Korea, in 1981 and 1966 respectively. He is a professor at Department of Computer Engineering, Hallym University, Korea. His research interests include computer network and e-learning. 
International Journal of Multimedia and Ubiquitous Engineering Vol. 9, No. 11 (2014)

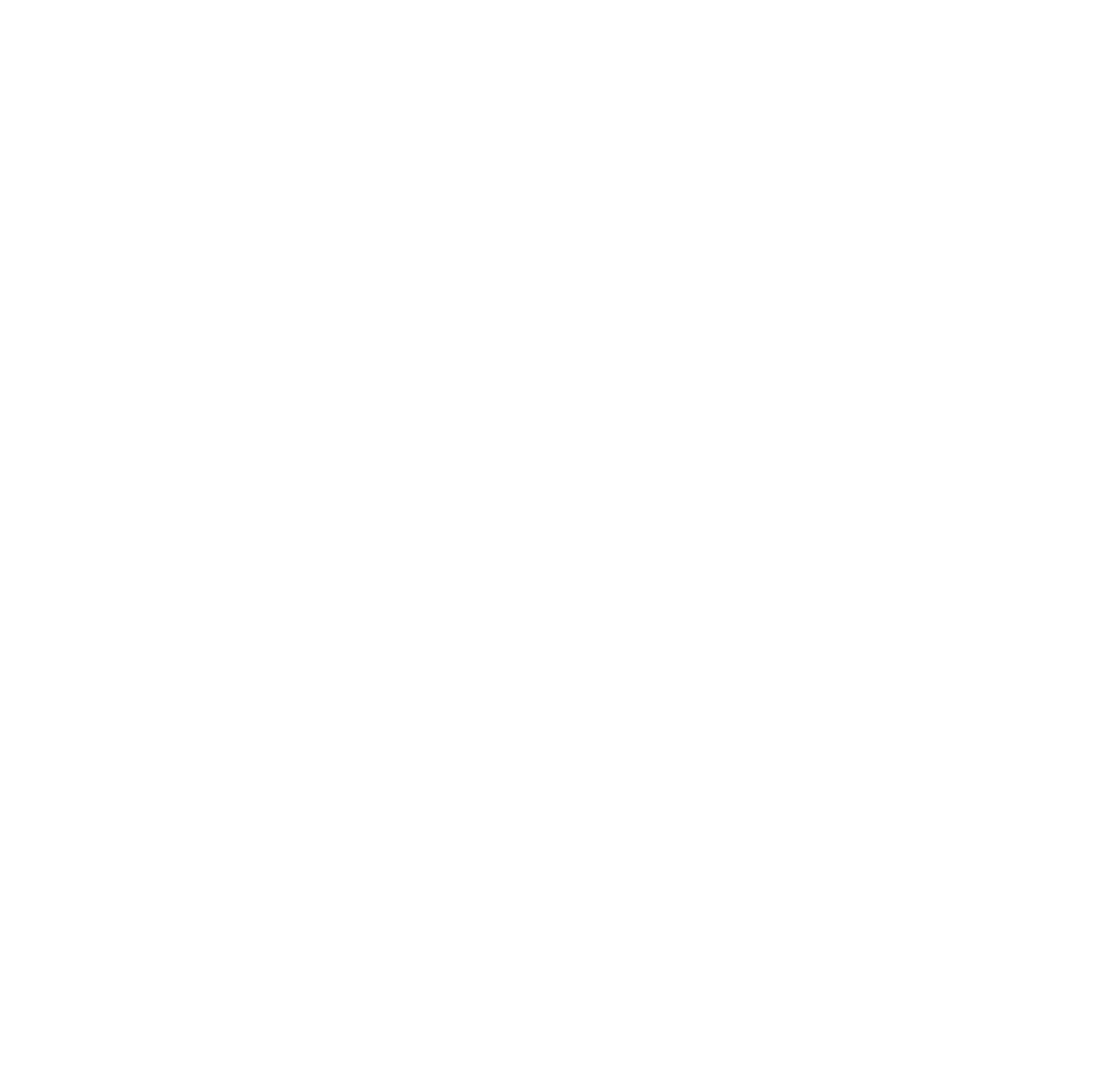

